\title{
A Survey on Identity-Based Proxy-Oriented Data Uploading and Remote Data Integrity Checking in Public Cloud
}

\author{
Pallavi S. Kaulage ${ }^{1}$, S. N. Kini ${ }^{2}$ \\ ${ }^{1}$ Department of Computer Engineering, Jayawantrao Sawant College of Engineering Pune, Maharashtra, India \\ ${ }^{2}$ Professor, Department of Computer Engineering, Jayawantrao Sawant College of Engineering Pune, Maharashtra, India
}

\begin{abstract}
More customers might want to store their information to PCS (open cloud servers) alongside the quick improvement of distributed computing. New security issues must be settled keeping in mind the end goal to help more customers process their information out in the open cloud. At the point when the customer is limited to get to PCS, he will designate its proxy to process his information and transfer them. Then again, remote information honesty checking is likewise an essential security issue out in the open distributed storage. It makes the customers check whether their outsourced information is kept in place without downloading the entire information. From the security issues, we propose a novel proxy arranged information transferring and remote information respectability checking model in personality based open key cryptography: IDPUIC (personality based proxy situated information transferring and remote information respectability checking in broad daylight cloud). We give the formal definition, framework model and security display. At that point, a solid ID-PUIC convention is composed by utilizing the bilinear pairings. The proposed ID-PUIC convention is provably secure in view of the hardness of CDH (computational Diffie-Hellman) issue. Our ID-PUIC convention is likewise proficient and adaptable. In view of the unique customer's approval, the proposed ID-PUIC convention can understand private remote information respectability checking, designated remote information respectability checking and open remote information trustworthiness checking.
\end{abstract}

Keywords: proxy, cloud, identity, data, checking

\section{Introduction}

Alongside the quick improvement of processing and correspondence strategy, a lot of information is produced. This enormous information needs more solid calculation asset and more prominent storage room. In the course of the most recent years, distributed computing fulfills the application necessities and becomes rapidly. Basically, it takes the information preparing as an administration, for example, capacity, registering, information security, and so on. By utilizing people in general cloud stage, the customers are eased of the weight for capacity administration, all inclusive information access with autonomous topographical areas, and so on. In this manner, more customers would like to store and process their information by utilizing the remote cloud registering framework.

Out in the open distributed computing, the customers store their enormous information in the remote open cloud servers. Since the put away information is outside of the control of the customers, it involves the security chances as far as classification, uprightness and accessibility of information and administration. Remote information uprightness checking is a primitive which can be utilized to persuade the cloud customers that their information are kept in place. In some extraordinary cases, the information proprietor might be confined to get to general society cloud server, the information proprietor will appoint the undertaking of information handling and transferring to the third party, for instance the proxy. On the opposite side, the remote information respectability checking convention must be productive with a specific end goal to make it reasonable for limit constrained end gadgets. Accordingly, based on character based open cryptography and proxy open key cryptography, we will examine ID-PUIC convention.

Openly cloud environment, most customers transfer their information to PCS and check their remote information's trustworthiness by Internet. At the point when the customer is an individual administrator, some useful issues will happen. In the event that the director is associated with being included into the business misrepresentation, he will be taken away by the police. Amid the time of examination, the supervisor will be limited to get to the system with a specific end goal to protect against agreement. Be that as it may, the director's legitimate business will go on amid the time of examination. At the point when a huge of information is produced, who can help him prepare this information? On the off chance that these information can't be handled without a moment to spare, the chief will confront the lose of monetary intrigue. With a specific end goal to avert the case happening, the chief needs to appoint the proxy to prepare its information, for instance, his secretary. Be that as it may, the administrator won't trust others can play out the remote information uprightness checking. Open checking will bring about some threat of releasing the protection. For instance, the put away information volume can be recognized by the malignant verifiers. At the point when the transferred information volume is classified, private remote information honesty checking is vital. In spite of the fact that the secretary can prepare what's more, transfer the information for the administrator, regardless he can't check the director's remote information honesty unless he is designated by the director. We call the secretary as the proxy of the administrator. 


\section{International Journal of Science and Research (IJSR) \\ ISSN (Online): 2319-7064}

Index Copernicus Value (2015): 78.96 | Impact Factor (2015): 6.391

In PKI (open key foundation), remote information honesty checking convention will play out the endorsement administration. At the point when the director assigns a few substances to play out the remote information respectability checking, it will bring about significant overheads since the verifier will check the authentication when it checks the remote information honesty. In PKI, the significant overheads originate from the overwhelming declaration confirmation, authentications era, conveyance, denial, recharges, and so on. In broad daylight distributed computing, the end gadgets may have low calculation limit, for example, cell phone, ipad, and so on. Personality based open key cryptography can take out the confounded testament administration. Keeping in mind the end goal to build the productivity, identity-based proxy arranged information transferring and remote information uprightness checking is more appealing. In this way, it will be exceptionally important to concentrate the ID-PUIC convention.

\section{Proxy Cryptography}

In an proxy re-encryption conspire, an proxy can change over an encryption registered under Alice's open key into an encryption proposed for Bob. Such a plan can be utilized by Alice to briefly forward scrambled messages to Bob without giving him her mystery key. The principal property of proxy re-encryption plans is that the proxy is not completely trusted, i.e., it doesn't know the mystery keys of Alice or Bob and does not take in the plaintext amid the transformation. The proxy and Bob, nonetheless, are not permitted to conspire, along these lines it is typically expected that no less than one of the two is straightforward or that their agreement is preventable or perceptible by means of different means. Various proxy re-encryption conventions have been proposed with regards to open key encryption .The thought of proxy re-encryption to the region of Identity-Based

Encryption (IBE), in which senders encode messages utilizing the beneficiary's character (a string) as the general population key. For example, Charles could encode a message for Alice by simply utilizing her email address. Initially presented by Shamir in 1984 and after that acknowledged by Boneh-Franklin and by Cocks quite a long while later, character based encryption has demonstrated valuable in explaining a few key-appropriation issues, and has allowed the improvement of an assortment of novel cryptographic conventions, e.g., mystery handshakes , open key searchable encryption, CCA2-secure open key encryption, and computerized marks. The Boneh-Franklin plan is especially proficient, and has been for all intents and purposes conveyed. The Identity based proxy re-encryption (IB-PRE) plans permit an proxy to interpret an encryption under Alice's personality into one processed under Bob's character. The proxy utilizes proxy keys, or re-encryption keys, to play out the interpretation without having the capacity to take in the plaintext. Also, no data on the mystery keys of Alice and Bob can be reasoned from the proxy keys.

\section{Identity-based Public Key Cryptography}

Identity-based proxy re-encryption (IB-PRE) plans permit and proxy to interpret an encryption under Alice's personality into one processed under Bob's character. The proxy utilizes proxy keys, or re-encryption keys, to play out the interpretation without having the capacity to take in the plaintext. Also, no data on the mystery keys of Alice and Bob can be derived from the proxy keys. Our developments are good with existing Boneh-Franklin IBE organizations, and can be executed utilizing existing privileged insights and parameters.

Keep in mind that clients in an Identity-Based Encryption plot ask for keys from a trusted gathering known as a Private Key Generator (PKG). In this way, on a fundamental level, it is conceivable that proxy keys could be produced by the PKG specifically. Be that as it may, we completely avoid this probability and we concentrate just on plans where singular clients assign their own unscrambling rights, without the contribution of the Private Key Generator. This is for hypothetical and useful reasons: (1) From a hypothetical perspective, having the $\mathrm{PKG}$, or some other trusted gathering, producing the proxy keys makes the issue of discovering IB-PRE plans very unchallenging given earlier workmanship, (2) from a viable perspective, it is unmistakably undesirable to have the PKG required in the era of proxy keys. It would constitute an extensive bottleneck in numerous applications, it would drive the PKG to be on-line and accessible notwithstanding amid the era of proxy keys (other than IBE keys), and, in specific applications, it would make the PKG obligated for making (conceivably undesirable) unscrambling rights.

Mambo and Okamoto proposed a procedure for assigning decoding rights in [16]. Burst, Bleumer and Strauss [3] later introduced the principal secure "nuclear" primitive: an Elgamal-based approach in which the proxy couldn't take in the message being prepared. Lamentably, the approach in [3] is natural bidirectional: a tainted proxy can re-encode cipher texts from Alice to Bob, as well as from Bob to Alice. Much more dreadful, conspiracy between the Proxy and "delegator" Alice could uncover the mystery key of "delegate" Bob. Jakobsson [15], and Zhou, Mars, Schneider and Redz [21] mostly tended to these worries by proposing a majority based convention which partitioned the proxy into numerous segments.

Later works have concentrated on the advancement of unidirectional proxy re-encryption plans, where plot between a delegator and the proxy does not bargain the delegate. Dodis and Ivan [12] understood a type of unidirectional proxy encryption by utilizing twofold encryption (or by part a solitary decoding key into two sections). Their approach allows a type of single-assignment proxy re-encryption at the point when gatherings hold preshared keys. Ateniese, Fu, Green and Hohenberger [1] proposed an enhanced, no interactive unidirectional plan which expelled the requirement for pre-shared keys and allowed discretionary designations.

Dodis and Ivan [12] additionally proposed an altogether different personality based proxy encryption plot in which 


\section{International Journal of Science and Research (IJSR) \\ ISSN (Online): 2319-7064 \\ Index Copernicus Value (2015): 78.96 | Impact Factor (2015): 6.391}

the PKG delegates decoding rights for all personalities in the framework (e.g., to give key escrow to law authorization). Such assignment is non-distinct, i.e., the PKG can't designate decoding rights for just a subset of personalities in the framework. This approach contrasts reasonably from our non-intuitive approach, where singular clients assign their unscrambling rights. At last, the Dodis/Ivan framework has noteworthy security suggestions: plot between the proxy and delegate brings about a framework wide trade off, permitting the colluders to remake the IBE ace mystery. As of late, Boneh, Goh and Matsuo [8] introduced a half breed type of proxy re-encryption in light of IBE. In such plans, the PKG plays out all assignments; along these lines clients can't perform disconnected ("non-intuitive") assignments and every designation requires an expensive online demand to the PKG. Besides, the Boneh-Goh Matsuo approach determines another private-key era calculation and it appears to be in this way inconsistent with existing IBE arrangements.

\section{Remote Data Integrity Checking}

In public cloud, remote data integrity checking is a critical security issue. Since the customers' monstrous information is outside of their control, the customers' information might be ruined by the vindictive cloud server paying little respect to deliberately or inadvertently. Keeping in mind the end goal to address the novel security issue, some proficient models are displayed.

In 2007, Ateniese et al. proposed provable data possession (PDP) worldview. In PDP demonstrate, the checker can check the remote information trustworthiness without recovering or downloading the entire information. PDP is a probabilistic evidence of remote information uprightness checking by testing arbitrary arrangement of squares from people in general cloud server, which radically decreases $\mathrm{I} / \mathrm{O}$ costs. The checker can perform the remote information uprightness checking by looking after little metadata. After that, some element PDP model and conventions are outlined.

Taking after Ateniese et al's. Spearheading work, numerous remote information trustworthiness checking models and conventions have been proposed.

In 2008, proof of retrievability (POR) plan was proposed by Shacham et al.. POR is a more grounded model which makes the checker checks the remote information honesty as well as additionally recovers the remote information. Numerous POR plans have been proposed. On a few cases, customer may appoint the remote information honesty checking errand to the outsider. In distributed computing, the outsider inspecting is imperative. By utilizing distributed storage, the customers can get to the remote information with autonomous geological areas. The end gadgets might be portable and constrained in calculation and capacity. In this manner, effective what's more, secure ID-PUIC convention is more reasonable for cloud customers furnished with versatile end gadgets.

From the part of the remote information honesty checker, all the remote information honesty checking conventions are grouped into two classes: private remote information trustworthiness checking what's more, open remote information trustworthiness checking. In the reaction checking period of private remote information honesty checking, a few private data is crucial. Despite what might be expected, private data is not required in the reaction checking of open remote information honesty checking. Extraordinarily, when the private data is designated to the outsider, the outsider can likewise play out the remote information honesty checking. For this situation, it is likewise called appointed checking.

\section{ID-PUIC Protocol Model}

In public cloud, the point concentrates on the personality based intermediary arranged information transferring and remote information uprightness checking. By utilizing character based open key cryptology, our proposed ID-PUIC convention is proficient since the authentication administration is wiped out. ID-PUIC is a novel intermediary situated information transferring and remote information honesty checking model out in the open cloud. We give the formal framework model and security display for ID-PUIC convention. At that point, in view of the bilinear pairings, we planned the main solid ID-PUIC convention. In the irregular prophet show, our outlined IDPUIC convention is provably secure. In view of the first customer's approval, our convention can understand private checking, designated checking and open checking

An ID-PUIC convention comprises of four diverse substances which are depicted underneath:

1) OriginalClient: a substance, which has gigantic information to be transferred to PCS by the designated intermediary, can perform the remote information trustworthiness checking.

2) PCS (Public Cloud Server): a substance, which is overseen by cloud specialist co-op, has huge storage room what's more, calculation asset to keep up the customers' information.

3) Proxy: a substance, which is approved to prepare the Original Client's information and transfer them, is chosen and approved by Original Client. At the point when Proxy fulfills the warrant $\mathrm{m}$ ! Which is marked and issued by Original- Customer, it can handle and transfer the first customer's information; else, it cannot play out the technique.

4) KGC (Key Generation Center): an element, while getting a character, it creates the private key which relates to the got character.

This solid ID-PUIC convention contains four strategies: Setup, Extract, Proxy-key era, TagGen, and Proof. In request to demonstrate the instinct of our development, the solid convention's design is portrayed in Figure 1. 


\section{International Journal of Science and Research (IJSR)

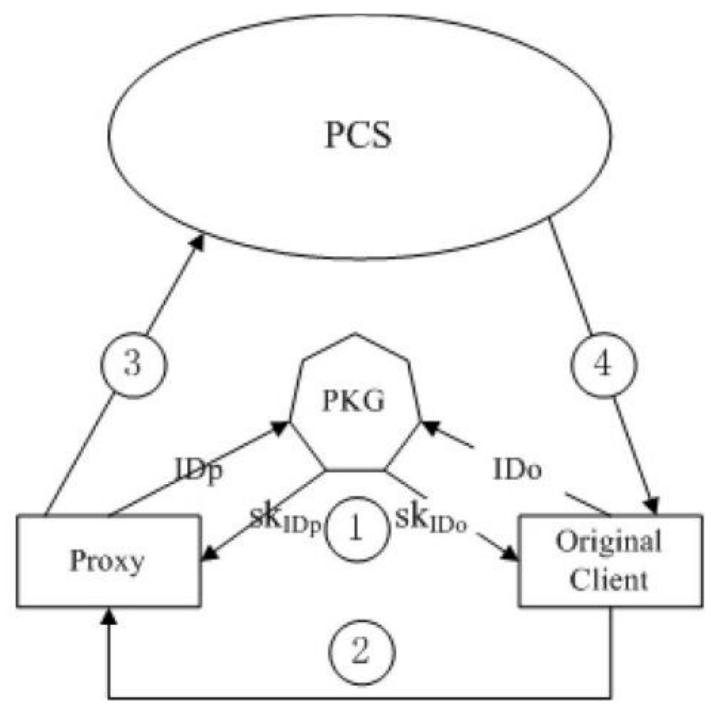

Fig. 1. Architecture of our ID-DPDP protocol.

To begin with, Setup is performed and the framework parameters are created. Based on the created framework parameters, alternate systems are executed as Figure 1. It is portrayed underneath:

1) In the stage Remove, when the element's character is information, KGC produces the substance's private key. Particularly, it can produce the private keys for the customer and the intermediary.

2) In the stage Proxy-key era, the first customer makes the warrant and aides the intermediary produce the intermediary key.

3) In the stage TagGen, when the information square is info, the intermediary creates the piece's tag furthermore; transfer square label sets to PCS.

4) In the stage Proof, the unique customer O collaborates with PCS. Through the cooperation, $\mathrm{O}$ checks its remote information honesty.

\section{Conclusion}

Roused by the application needs, this paper proposes the novel security idea of ID-PUIC in broad daylight cloud. The paper formalizes ID-PUIC's framework model and security display. At that point, the primary solid ID-PUIC convention is outlined by utilizing the bilinear pairings method. The solid ID-PUIC convention is provably secure and productive by utilizing the formal security verification and effectiveness examination. Then again, the proposed ID-PUIC convention can likewise acknowledge private remote information honesty checking, appointed remote information uprightness checking and open remote information honesty checking in light of the first customer's approval.

\section{References}

[1] Z. Fu, X. Sun, Q. Liu, L. Zhou, J. Shu, "Achieving efficient cloud search services: multi-keyword ranked search over encrypted cloud data supporting parallel computing," IEICE Transactions on Communications,vol. E98-B, no. 1, pp.190-200, 2015.

[2] Y. Ren, J. Shen, J. Wang, J. Han, S. Lee, "Mutual verifiable provable data auditing in public cloud storage," Journal of Internet Technology, vol. 16, no. 2, pp. 317-323, 2015.

[3] M. Mambo, K. Usuda, E. Okamoto, "Proxy signature for delegating signing operation”, CCS 1996, pp. 48C57, 1996.

[4] E. Yoon, Y. Choi, C. Kim, "New ID-based proxy signature scheme with message recovery", Grid and Pervasive Computing, LNCS 7861, pp. 945-951, 2013.

[5] B. Chen, H. Yeh, "Secure proxy signature schemes from the weil pairing”, Journal of Supercomputing, vol. 65, no. 2, pp. 496-506, 2013.

[6] X. Liu, J. Ma, J. Xiong, T. Zhang, Q. Li, "Personal health records integrity verification using attribute based proxy signature in cloud computing", Internet and Distributed Computing Systems, LNCS 8223, pp. 238-251, 2013.

[7] H. Guo, Z. Zhang, J. Zhang, "Proxy re-encryption with unforgeable reencryption keys", Cryptology and Network Security, LNCS 8813, pp. 20-33, 2014.

[8] E. Kirshanova, "Proxy re-encryption from lattices", PKC 2014, LNCS 8383, pp. 77-94, 2014.

[9] P. Xu, H. Chen, D. Zou, H. Jin, "Fine-grained and heterogeneous proxy re-encryption for secure cloud storage", Chinese Science Bulletin, vol.59, no.32, pp. 4201-4209, 2014.

[10] S. Ohata, Y. Kawai, T. Matsuda, G. Hanaoka, K. Matsuura, "Reencryption verifiability: how to detect malicious activities of a proxy in proxy re-encryption", CT-RSA 2015, LNCS 9048, pp. 410-428, 2015.

[11] G. Ateniese, R. Burns, R. Curtmola, J. Herring, L. Kissner, Z. Peterson, D. Song, "Provable data possession at untrusted stores”, CCS'07, pp. 598-609, 2007.

[12] G. Ateniese, R. DiPietro, L. V. Mancini, G. Tsudik, "Scalable and efficient provable data possession", SecureComm 2008, 2008.

[13] C. C. Erway, A. K"upc, "u, C. Papamanthou, R. Tamassia, "Dynamic provable data possession", CCS'09, pp. 213-222, 2009.

[14]E. Esiner, A. K"upc, "u, "O zkasap, "Analysis and optimization on FlexDPDP: a practical solution for dynamic provable data possession", Intelligent Cloud Computing, LNCS 8993, pp. 65-83, 2014.

[15] E. Zhou, Z. Li, "An improved remote data possession checking protocol in cloud storage", Algorithms and Architectures for Parallel Processing, LNCS 8631, pp. 611-617, 2014.

[16]H. Wang, "Proxy provable data possession in public clouds," IEEE Transactions on Services Computing, vol. 6 , no. 4, pp. 551-559, 2013

[17]H. Wang, "Identity-based distributed provable data possession in multicloud storage", IEEE Transactions on Services Computing, vol. 8, no. 2, pp. 328-340, 2015.

[18]H. Wang, Q. Wu, B. Qin, J. Domingo-Ferrer, "FRR: Fair remote retrieval of outsourced private medical records in electronic health networks", Journal of Biomedical Informatics, vol. 50, pp. 226-233, 2014.

[19]H. Wang, "Anonymous multi-receiver remote data retrieval for pay-tv in public clouds", IET Information Security, vol. 9, no. 2, pp. 108-118, 2015.

[20]H. Shacham, B. Waters, "Compact proofs of retrievability”, ASIACRYPT 2008, LNCS 5350, pp. 90107,2008 


\section{International Journal of Science and Research (IJSR) \\ ISSN (Online): 2319-7064 \\ Index Copernicus Value (2015): 78.96 | Impact Factor (2015): 6.391}

[21]Q. Zheng, S. Xu, "Fair and dynamic proofs of retrievability", CODASPY' 11, pp. 237-248, 2011.

[22] D. Cash, A. K"upc, "u, D. Wichs, "Dynamic proofs of retrievability via oblivious ram", EUROCRYPT 2013, LNCS 7881, pp. 279-295, 2013.

[23] J. Zhang, W. Tang, J. Mao, "Efficient public verification proof of retrievability scheme in cloud", Cluster Computing, vol. 17, no. 4, pp. 1401-1411, 2014.

[24] J. Shen, H. Tan, J. Wang, J. Wang, S. Lee, “A novel routing protocol providing good transmission reliability in underwater sensor networks", Journal of Internet Technology, vol. 16, no. 1, pp. 171-178, 2015.

[25] T. Ma, J. Zhou, M. Tang, Y. Tian, Al-dhelaan A., Alrodhaan M., L. Sungyoung, "Social network and tag sources based augmenting collaborative recommender system", IEICE Transactions on Information and Systems, vol.E98-D, no.4, pp. 902-910, 2015.

[26] K. Huang, J. Liu, M. Xian, H. Wang, S. Fu, "Enabling dynamic proof of retrievability in regenerating-codingbased cloud storage", ICC 2014, pp.712-717, 2014.

[27]C. Wang, Q. Wang, K. Ren, W. Lou, "Privacypreserving public auditing for data storage security in cloud computing", INFOCOM 2010, pp. 1-9, 2010.

[28] Q. Wang, C. Wang, K. Ren, W. Lou, J. Li, "Enabling public auditability and data dynamics for storage security in cloud computing", IEEE Transactions on Parallel And Distributed Systems, vol. 22, no. 5, pp. 847-859, 2011.

[29] C. Wang, Q. Wang, K. Ren, N. Cao, W. Lou, "Toward secure and dependable storage services in cloud computing," IEEE Transactions on Services Computing, vol. 5, no. 2, pp. 220-232, 2012.

[30] Y. Zhu, G. Ahn, H. Hu, S. Yau, H. An, S. Chen, "Dynamic Audit Services for Outsourced Storages in Clouds," IEEE Transactions on Services Computing, vol. 6, no. 2, pp. 227-238, 2013.

[31] O. Goldreich, "Foundations of cryptography: basic tools", Publishing House of Electronics Industry, Beijing, pp. 194-195, 2003.

[32] D. Boneh, B. Lynn, H. Shacham, "Short signatures from the weil pairing", ASIACRYPT 2001, LNCS 2248, pp. 514-532, 2001.

[33] D. Boneh, M. Franklin, "Identity-based encryption from the weil pairing", CRYPTO 2001, LNCS 2139, pp. 213229, 2001.

[34] A. Miyaji, M. Nakabayashi, S. Takano, "New explicit conditions of elliptic curve traces for fr-reduction", IEICE Transactions Fundamentals, vol. 5, pp. 12341243, 2001.

[35] C. Research, "SEC 2: Recommended elliptic curve domain parameters", http://www.secg.org/collateral/sec final.pdf

[36] The GNU multiple precision arithmetic library (GMP). Available: http://gmplib.org/.

[37] The pairing-based cryptography library (PBC). Available: http://crypto.stanford.edu/pbc/howto.html.

[38]Lynn B., "On the implementation of pairing-based cryptosystems", Ph.D. dissertation, http://crypto.stanford.edu/pbc/thesis.pdf, Stanford University, 2008. 